\title{
Atomic-Orbital Close-Coupling Calculations of Electron Capture from Hydrogen Atoms Into Highly Excited Rydberg States of Multiply Charged Ions
}

\author{
Katharina Igenbergs ${ }^{\mathrm{a}}$, Markus Wallerberger ${ }^{\mathrm{a}}$, Josef Schweinzer ${ }^{\mathrm{b}}$ \\ and Friedrich Aumayr ${ }^{b}$ \\ ${ }^{a}$ Institute of Applied Physics, Vienna University of Technology, Association ÖAW-EURATOM, \\ Wiedner Hauptstr.8-10/E134, 1040 Vienna, Austria \\ ${ }^{b}$ Max-Planck-Institut für Plasmaphysik, EURATOM Association, Boltzmannstr.2, 85748 Garching, Germany
}

\begin{abstract}
Collisions of neutral hydrogen atoms with multiply charged ions have been studied in the past using the semiclassical atomic-orbital close-coupling method. We present total and state-resolved cross sections for charge exchange as well as ionization. The advent of supercomputers and parallel programming facilities now allow treatment of collision systems that have been out of reach before, because much larger basis sets involving high quantum numbers are now feasible
\end{abstract}

Keywords: electron capture, charge transfer, cross section, ion-atom collision, atomic-orbital close-coupling.

PACS: $31.15 x r, 52.20 . \mathrm{Hv}, 34.50 . \mathrm{Fa}, 52.75 .-\mathrm{d}, 52.25 . \mathrm{Vy}$

\section{MOTIVATION}

Collisions between neutral hydrogen isotopes and multiply charged ions resulting in charge exchange (CX) and ionization (ION) have been the subject of a large number of studies in the past [1]. Major motivation arises from thermonuclear fusion research since these kinds of cross sections are needed for a variety of applications, in particular charge exchange recombination spectroscopy (CXRS) [2]. This diagnostic technique relies on the injection of fast neutral hydrogen (deuterium) atoms into the hot region of the plasma (e.g., by using the neutral heating beam). Through collisions with multiply charged impurity ions, the electron from the hydrogen can be captured into excited Rydberg states of the ion. The subsequent emission of photons with characteristic wavelengths and intensities, and Doppler broadening and shifts allows determination of the density and temperature of the plasma ions as well as the direction and the velocity of the plasma flow [2].

\section{THEORETICAL METHOD - ATOMIC- ORBITAL CLOSE-COUPLING}

We have applied the atomic-orbital close-coupling (AOCC) algorithm with both hydrogen-like states and pseudostates that represent the continuum $[1,3]$. The wavefunction of the active electron in the target and the projectile potentials is expanded in terms of basis states. Convergence of the solution of the closecoupling equations with increasing basis set size is achieved by systematically increasing the number of basis states on each center. The time-dependent Schrödinger equation is now solved in the truncated Hilbert space (i.e., the subspace spanned by the basis functions in matrix formulation) [4]. Therefore we need to calculate overlap and coupling matrix elements that can be subsumed under the term exchange matrix elements or exchange integrals.

\section{Computational Challenges \& Parallelization}

The calculation of these matrix elements becomes more and more complex and time consuming when approaching high quantum numbers. Generally, we solve these exchange integrals using the Fouriertransform method [4]. We significantly optimized the respective numerical routines and are thus now able to calculate results using even larger basis sets up to much higher principal quantum numbers $n$, angular momentum quantum numbers $l$, and magnetic quantum numbers $m$ [5]. 
Additionally, we introduced 'non-coupling' basis states where the interaction with the other center is neglected. Such states can be used below $\sim 10$ $\mathrm{keV} / \mathrm{amu}$ and above $\sim 100 \mathrm{keV} / \mathrm{amu}$. These noncoupling channels are centered on the hydrogen atom, where a large number of excitation and ionization channels can easily be included. In the intermediate energy region, however, these channels also interact significantly with the basis states on the ion center. Therefore, such an approximation cannot be made here. Nevertheless, this approximation is very useful when analyzing the behavior of AOCC calculations with different basis sets with respect to convergence of results. We focus our efforts mainly on impact energies that are of interest in neutral beam diagnostics of hot nuclear fusion plasmas (i.e., roughly between 10 and $100 \mathrm{keV} / \mathrm{amu})$. In this region, all inelastic channels are in competition and therefore the AOCC approach faces great challenges especially when treating highly charged ions such as $\mathrm{Ar}^{\mathrm{q}^{+}}$with $\mathrm{q} \approx 15$ 18.

\section{RESULTS}

We present total CX and ION cross sections, as well as state-resolved $\mathrm{CX}$ cross sections. The total cross sections are compared to data from the literature and the $n$ - and $n l$-resolved cross sections are used to calculate emission cross sections and effective emission coefficients. The latter are the quantities that are actually needed in the analysis of CXRS data for diagnosing thermonuclear plasmas. The effective emission coefficient is defined as the emission cross section averaged over a velocity distribution,

$$
\begin{aligned}
& q_{n_{i} \rightarrow n_{f}}^{\text {eff }}=\iiint d^{3} \vec{v} \sigma_{n_{i} \rightarrow n_{f}}^{\text {emi }}\left(\left|\vec{v}-\vec{v}_{\text {beam }}\right|\right)\left|\vec{v}-\vec{v}_{\text {beam }}\right| f(|\vec{v}|) \\
& f(\vec{v})=f(|\vec{v}|)=f(v)=c e^{-v^{2} / w^{2}} \\
& c \equiv\left(\frac{m}{2 \pi k_{B} T}\right)^{3 / 2}, w^{2} \equiv \frac{2 k_{B} T}{m}
\end{aligned}
$$

where $f(v)$ is the Maxwellian speed distribution, $k_{B}$ the Boltzmann constant, $T$ the plasma temperature, and $m$ the mass of the impurity ion. Using SI units throughout, the effective emission coefficients can be given in $\mathrm{cm}^{3} \mathrm{~s}^{-1}$.

$$
B e^{4+}+H(n=1,2)
$$

Although a fairly light ion and by no means a new collisional system to be studied, the impact of fully stripped beryllium ions on neutral hydrogen in ground an excited states has recently received increased interest. With the decision to build ITER, fusion laboratories around the world have strongly focused their experimental activities on ITER-relevant research. In the course of these developments, the inner wall of the Joint European Torus (JET) is going to be changed to resemble the one planned for ITER. From 2011 onwards tungsten will be used in the divertor and beryllium for the first wall [6] of JET. Plasma wall interaction processes will lead to a high concentration of beryllium ions in the JET plasma. Therefore CXRS based on Be line emission will be an important and powerful diagnostic tool.

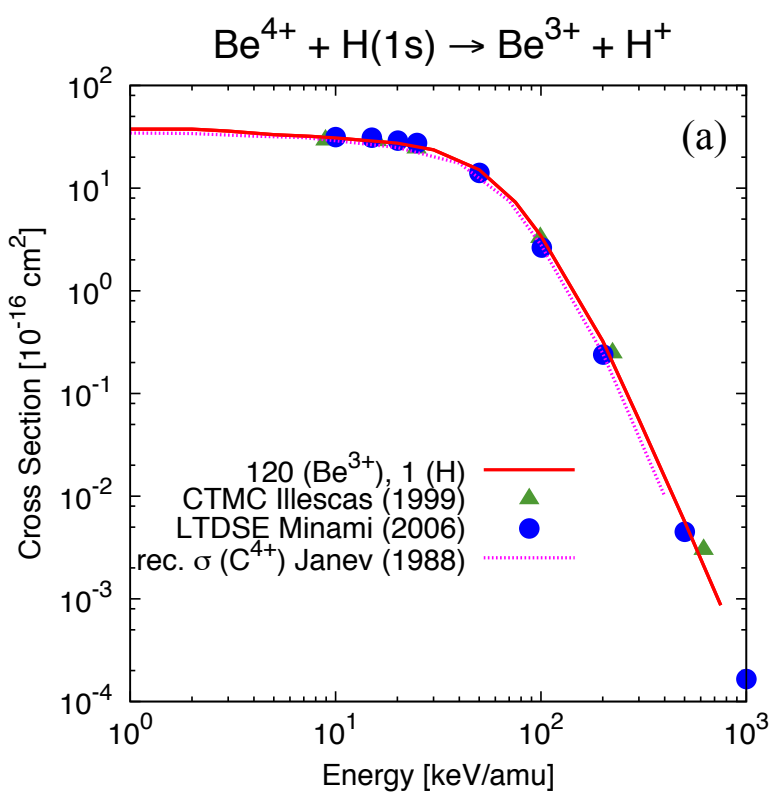

FIGURE 1. Total charge exchange cross sections for (a) $\mathrm{Be}^{4+}+\mathrm{H}(1 \mathrm{~s})$ in comparison with data from [7], [8], [9] and for (b) $\mathrm{Be}^{4+}$ $+\mathrm{H}(\mathrm{n}=2)$ in comparison with data from [10], [11], [12]. A more elaborate analysis of these results can be found in [3]. 

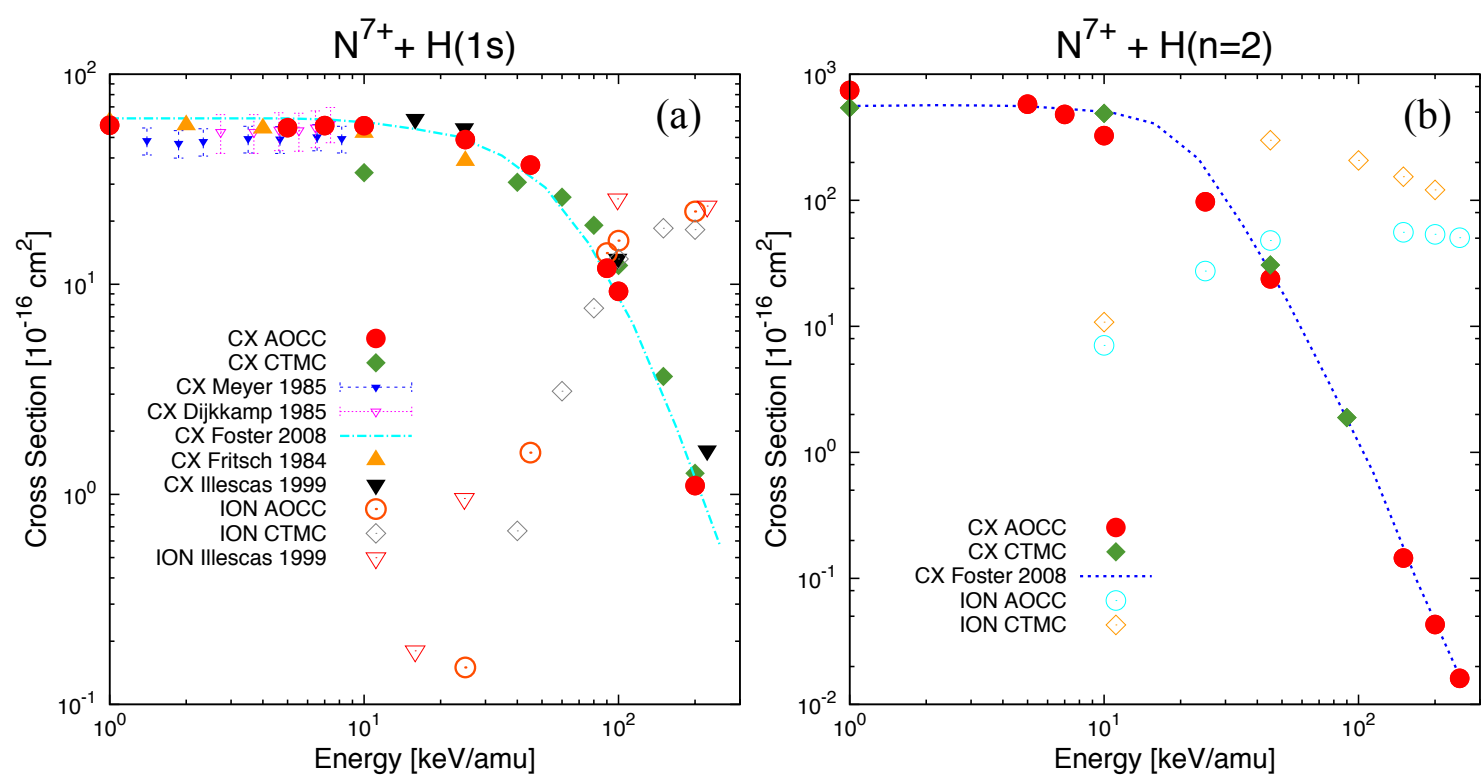

FIGURE 2. Total charge exchange cross sections and ionization cross sections calculated by AOCC and CTMC from [14] for (a) $\mathrm{N}^{7+}+\mathrm{H}(1 \mathrm{~s})$ in comparison with data from [15], [8], and for (b) $\mathrm{N}^{7+}+\mathrm{H}(\mathrm{n}=2)$ in comparison with data from [16].

Fig. 1 shows total charge exchange cross sections for $\mathrm{Be}^{4+}$ impact on both $\mathrm{H}(1 \mathrm{~s})$ (Fig.1a) and excited $\mathrm{H}(\mathrm{n}=2)$ (Fig.1b). The latter cross sections are statistically averaged cross sections for $\mathrm{H}(2 \mathrm{~s}), \mathrm{H}\left(2 \mathrm{p}_{0}\right), \mathrm{H}\left(2 \mathrm{p}_{1}\right)$, and $\mathrm{H}\left(2 \mathrm{p}_{-1}\right)$ [3]. Our data [3] for the H(1s) target show very good agreement with data calculated by Minami et al. in the lattice time-dependent Schrödinger equation (LTDSE) approach [7], classical trajectory Monte Carlo (CTMC) cross sections from Illescas and Riera [8], and recommended cross sections for $\mathrm{C}^{4+}$ ions from Janev et al. [9]. In the case of the $\mathrm{H}(\mathrm{n}=2)$ target, CTMC cross section from Errea et al. [10], LandauZener data from Casaubon [11], and another set of CTMC calculations from Hoekstra et al. [12] are shown in Fig.1a for comparison. In this case the agreement is not as excellent as in the $\mathrm{H}(1 \mathrm{~s})$ case, but is nevertheless quite satisfactory.

$$
\mathbf{N}^{7+}+\mathbf{H}(\mathrm{n}=1,2)
$$

Nitrogen is used as a seeding impurity for radiative plasma edge cooling at ASDEX Upgrade [13]. In order to limit the heat flux on the small wetted area of the divertor plates, radiating impurities can be puffed into the plasma chamber. When they get ionized and excited by electron (and ion) impact in the plasma edge, they distribute a fraction of this heat flux to larger areas by radiation, thus reducing the power load on divertor components and cooling the outermost plasma regions. At ASDEX Upgrade, the use of nitrogen seeding is accompanied by improved energy confinement due to higher plasma temperatures that more than compensates the negative effect of plasma dilution by nitrogen. Therefore, it recently became an interesting species for CXRS. In [14], we presented AOCC and CTMC charge exchange as well as ionization cross sections. For the latter, we had to include 34 unbound pseudostates on the hydrogen center. Fig.2 shows that these AOCC ION cross sections agree very well with CTMC [14]. The total $\mathrm{CX}$ cross sections are compared to earlier AOCC calculations including pseudostates $(\mathrm{AO}+)$ [15], CTMC data [8], as well as scaled cross sections calculated using the ADAS315 routine [16] included in the Atomic Data and Analysis Structure (ADAS) software and data package.

\section{TOWARD HEAVY ION IMPACT CROSS SECTIONS}

The major challenges in trying to expand the AOCC method beyond its present boundaries are twofold. On the one hand, computational times rise rapidly for larger basis sets involving high quantum numbers $n, l$, $m$ required to treat capture to more highly charged and heavier ions and on the other, numerical instabilities when treating high $n$ basis functions needs to be carefully addressed. For example, $\mathrm{Ne}^{10+}$ has a major capture channel for $n=6$ and the visible lines in the $\mathrm{Ne}^{9+}$ spectrum originate from even higher $n$-shells $(n=10,11$, or 12$)$. For fully stripped $\mathrm{Ar}^{18+}$ ions the major capture channel rises to $n=10$ and the visible lines come from transitions starting at $n=14,15,16$, and 17. 


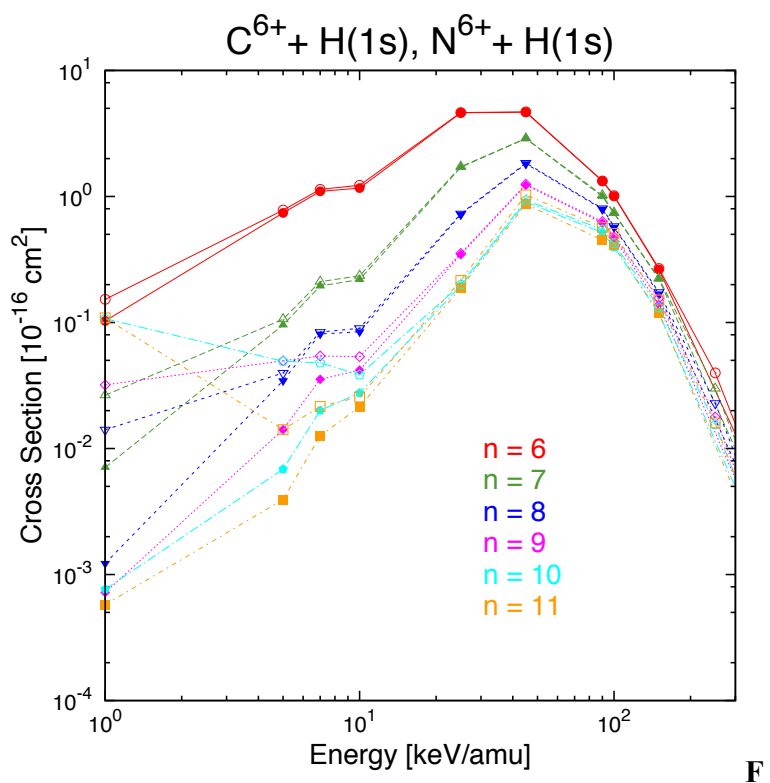

FIGURE 3. $n$-resolved cross sections of $\mathrm{C}^{6+}+\mathrm{H}(1 \mathrm{~s})$ (closed symbols) in comparison to $\mathrm{N}^{6+}+\mathrm{H}(1 \mathrm{~s})$ (open symbols).

For these noble gas ions, the fractional abundances of not fully-stripped ions at plasma temperatures is non-negligible. In single-electron transfer collisions, the AOCC method treats all passive electrons as perturbations to the potential of the active electron on the respective collision center. This means that elaborate potentials need to be found resulting in much more complex structures of the matrix elements. It is, nevertheless, a reasonable assumption that the influence of closely bound core electrons on the active electron that captures into very high $n$-shells is negligible. We therefore conducted a study of $\mathrm{C}^{6+}+$ $\mathrm{H}(1 \mathrm{~s})$ in comparison to $\mathrm{N}^{6+}+\mathrm{H}(1 \mathrm{~s})$. The main capture channel of the active electron is $n=4$, which is of course much lower than in the case of highly charged $\mathrm{Ne}$ or Ar ions. One would therefore expect differences in the cross sections as a result of the perturbed potential to be more pronounced. Fig.3 shows nresolved CX cross sections of both collisional systems in comparison. At low energies, well below 10 $\mathrm{keV} / \mathrm{amu}$, there is a certain difference, but when looking at fusion relevant energy regions it becomes obvious that the cross sections do not deviate from each other anymore [14].

\section{ACKNOWLEDGEMENTS}

Katharina Igenbergs is a fellow of the Friedrich Schiedel Foundation for Energy Technology. Furthermore, her attendance of CAARI 2010 was also subsidized by a Mariette-Blau mobility scholarship of Vienna University of Technology. The results presented in this paper have been achieved in part using the Vienna Scientific Cluster (VSC).

This work, supported by the European Communities under the Contract of Association between EURATOM and the Austrian Academy of Sciences, was carried out within the framework of the European Fusion Development Agreement. The views and opinions expressed herein do not necessarily reflect those of the European Commission.

\section{REFERENCES}

1. W. Fritsch and C.D. Lin, Phys. Reports 202, 1-97 (1991).

2. R. Isler, Plasma Phys. Contr. Fusion 36, 171-208 (1994).

3. K. Igenbergs, J. Schweinzer, F. Aumayr, J. Phys. B 42, 235206 (2009)

4. R. Shakeshaft, J. Phys. B 8, L134-L136 (1975)

5. M. Wallerberger, K. Igenbergs, J. Schweinzer, F. Aumayr, accepted by Computer Phys. Comm. (2010)

6. J. Paméla, G.F. Matthews, V. Philipps, R. Kamendje, J. Nucl. Mat. 363-365, 1-11 (2007)

7. T. Minami, M. Pindzola, D. Schultz, J. Phys. B 39, 28772891 (2006)

8. C. Illescas, A. Riera, Phys. Rev. A 60, 4546-4560 (1999)

9. R. Janev, R. Phaneuf, H. Hunter, At. Data Nucl. Data Tables 40, 249-281 (1988)

10. L.F. Errea, C. Harel, H. Jouin, L. Mendez, B. Pons, Riera A., J. Phys. B 31, 3527-3545

11. J.I. Casaubon, Phys. Rev. A 48, 3680-3683 (1993)

12. R. Hoekstra, H. Anderson, F. Blieck, M. von Hellermann, C. Maggi, R.E. Olson, H. Summers, Plasma Phys. Contr. Fusion 40, 1541-1550 (1998)

13. A. Kallenbach et al., Plasma Phys. Contr. Fusion 52, 055002 (2010)

14. K. Igenbergs et al, submitted to J. Phys. B (2010)

15. W. Fritsch \& C.D. Lin, Phys. Rev. A 29, 3039-3051 (1984)

16. A. Foster, On the Behaviour and Radiating properties of Heavy Elements in Fusion Plasmas, PhD thesis, University of Strathclyde (2008) 